\title{
Future challenges and perspectives for applying microbial biotechnology in sustainable agriculture based on a better understanding of plant-microbiome interactions
}

\author{
J. M. Barea \\ Departamento de Microbiología del Suelo y Sistemas Simbióticos, Estación Experimental del Zaidín, CSIC. \\ Prof. Albareda 1, 18008 Granada, Spain.Corresponding author: jmbarea@eez.csic.es
}

\begin{abstract}
An intensive agricultural production is necessary to satisfy food requirements for the growing world population. However, its realization is associated with the mass consumption of non-renewable natural resources and with the emission of greenhouse gases causing climate changes. The research challenge is to meet sustainable environmental and economical issues without compromising yields. In this context, exploiting the agroecosystem services of soil microbial communities appears as a promising effective approach. This chapter reviews the research efforts aimed at improving a sustainable and healthy agricultural production through the appropriate management of soil microorganisms. First, the plant-associated microbiome is briefly described. Then, the current research technologies for formulation and application of inocula based on specific beneficial plant-associated microbes are summarized. Finally, the perspectives and opportunities to manage naturally existing microbial populations, including those non-culturable, are analyzed. This analysis concerns: (i) a description of the already available, culture-independent, molecular techniques addressed at increasing our understanding of root-microbiome interactions; (ii) how to improve the ability of soil microbes for alleviating the negative impacts of stress factors on crop productivity; and (iii) whether plants can structure their rootassociated microbial communities and, leading on from this, whether the rhizosphere can be engineered (biased) to encourage beneficial organisms, while prevent presence of pathogens.
\end{abstract}

Keywords: Sustainable food production, microbial services, root-microbiome interactions, "omics"- driven microbial ecology, biased rhizospheres

\section{Introduction}

According to information from specialized sources, demand for agricultural production is expected to increase by at least $70 \%$ by 2050 . At the same time, people are becoming aware that sustainable agricultural practices are fundamental to meet the future world's agricultural demands (Altieri, 2004). This is why modern agriculture is being implemented on a global scale and diverse research 
approaches are being undertaken addressed to meet environmental and economical sustainability issues, trying to save at most as possible usage of non-renewable natural resources. A recommended approach is that based on exploiting the role of soil microbial communities for a sustainable and healthy crop production, while preserving the biosphere. Actually, soil microorganisms play fundamental roles (microbial services) in agriculture mainly by improving plant nutrition and health, as well as soil quality (Barea et al., 2013a; Lugtenberg, 2015). Accordingly, several strategies for a more effective exploitation of beneficial microbial services, as a lowinput biotechnology, to help sustain environmentally friendly agro-technological practices have been, and are being, proposed. The final goal is to optimize the role of the root-associated microbiome in nutrient supply and plant protection (Raaijmakers and Lugtenberg, 2013). Since the interactions between microbial communities and crops are influenced by diverse ecological factors and agronomic managements, the impact of environmental stress factors must be considered, particularly in the current scenario of global change, as they affect a proper management of the crop-microbiome interactions (Zolla et al., 2013).

This article is an overview of those strategies addressed to an effective exploitation of beneficial microbial services in sustainable agriculture. After analyzing the problematic, challenges and opportunities, this study focuses on describing the role of the plant-associated microbiome and their feasible managements. Finally, a fundamental part of this review is devoted to discuss the future perspectives and opportunities related to: (i) improving our understanding of the plant-microbiome interactions; (ii) enhancing the ability of soil microbes for stress alleviation in crops; (iii) learning whether the rhizosphere can be breaded or engineered to enrich beneficial microbial functions, leading to the concept/ action of a "biased" rhizosphere.

\section{Problems, challenges and opportunities}

Scientists are aware on addressing their research efforts to face a critical problem derived from the need to feed a growing, and more and more urbanized, world population. Actually, 10 billion people are expected to inhabit the Planet by 2050, with a particular incidence in developing countries. Consequently, many challenges arise, basically, the need to produce more food, fiber and bio-energy, while preserving the biosphere. An intensive agricultural production appears necessary, however these practices imply the mass consumption of non-renewable natural resources, such as fossil fuel and other energy sources, water, agricultural soil, rock phosphate reserves, etc. In addition, both the energy intensive industrial processes for the production of fertilizers, and the runoff or leaching of soluble nutrients from the applied agrochemicals into the aquatic systems, are sources of environmental contamination (Browne et al., 2013).

Besides, intensive agriculture is known to cause an increase in the production of "greenhouse gases", thereby rising Earth's temperature, thus affecting biosphere stability (Duarte et al., 2006). Consequently, diverse types of stress situations are generated by intensive agricultural practices, all of them impacting on the functionality/productivity of both agricultural systems and natural ecosystems, and limit the services that these are able to provide. The responsible stress factors include salinity, drought, nutrient deficits, contamination, soil erosion, diseases, pests, plant invasions, etc. In addition, the application of agrochemicals to control biotic stresses and nutrient deficiencies provokes environmental contamination and may threat human health. In summary, the above indicated ecological constraints impact on agro-ecosystems and cause agricultural and forest productivity losses, soil erosion, water deficit, 
biodiversity losses, landscape fragmentation, etc. (Vitousek et al., 1997).

Agricultural practices are currently implemented on a global scale and different approaches are being addressed to meet sustainable environmental and economical developments with the final aims of maintaining yield while preserving the biosphere. Altieri (2004) defines "sustainable development" as the result of the intersections among three primary factors: environment, society and economy, which in turn interact between each two of them. Therefore, the intersection economy-environment (agro-ecology), environment-society (environmental awareness), and society-economy (life standard), finally determines the concept/action of "sustainable development". In a sustainability context, a key issue is maintaining the quality of the soil, a non-renewable resource, which exerts many environmental and social functions some of them are driven by soil microbes (Zacarini et al., 2013). A target in sustainability is to find out efficient methods for recycling nutrients, controlling pest and pathogens, and for alleviating the negative impact of abiotic stress factors, fundamental issues for human life and for the sustainability of global ecosystems. These activities are typical microbial services, which can be exploited after an appropriate management of beneficial microorganisms and their functions (Zolla et al., 2013). Accordingly, the role and management of the root-associated microbiome, essential to meet both economically and ecologically sustainable issues, is analyzed first in this article.

\section{Types of root-associated microorganisms and strategies for their management}

\subsection{The plant-associated microbiome}

Soil microbes are recognized as a relevant component within the diverse interacting factors responsible for the environmental quality needed for a sustainable healthy food production. Microorganisms are attracted to, and maintained at, rhizosphere microhabitats by the rhizodeposit pools (Hirsch et al., 2013b). The soil microbiome comprises diverse types of organisms, but bacteria, fungi, and archaea are those receiving by far more attention in soil microbiology studies (Spence and Bais, 2013). Around $10^{9}$ microbial cells per $\mathrm{g}$ of soil have been recognized. These exhibit a great diversity level, reaching about $10^{6}$ taxa. However, only $1 \%$ of microorganisms living in the bulk soil, and $10 \%$ of those inhabiting plant-influenced zones, are able to grow in standard culture media while the rest remains as unculturable microbes, but detectable using molecular-based approaches, as discussed later (Barret et al., 2013).

The plant-associated prokaryotic bacteria and the eukaryotic fungi have a great variety of trophic/living habits whose saprophytic or symbiotic relationships with the plant could be either detrimental or beneficial. Most of these microbes remain in the rhizospheric soil or rhizoplane, but a small subpopulation of them, designated as "endophytes", is able to penetrate and live within plant tissues (Porras-Alfaro and Bayman, 2011; Hardoim et al., 2013; Nafalnova et al., 2013; Brader et al., 2014; Mercado-Blanco, 2015). The endophytes escape from immune plant responses and colonize, without causing symptoms of disease, different plant parts (roots, shoots, leaves or fruits), in different compartments of the plant apoplast (intercellular spaces and xylem vessels) and, in cases, inside the plant cells. Some endophytes affect plant growth and plant responses to pathogens, herbivores, and environmental changes, or produce important secondary metabolites. Most endophytes are unculturable, therefore the analysis of their diversity and the molecular basis of their interactions with the plant are revealed by using molecular approaches. Strictly speaking, other microbial groups that colonize 
plant tissues, i. e. mycorrhizal fungi, rhizobia, some pathogens and other, are actually endophytes, but they are considered separately from the core group of "endophytes", as involved in either nutrient transfer from sources outside the root, i. e. soil or atmosphere, or cause disease symptoms in their host plant.

\subsection{Beneficial rhizosphere microorganisms}

Beneficial saprophytic rhizosphere microbes improve plant performance acting as: (i) decomposer of organic substances (detritus); (ii) plant growth promoting rhizobacteria (PGPR); or (iii) antagonists of plant pathogens. The PGPR are known to participate in many important ecosystem processes, such as the biological control of plant pathogens and nutrient cycling. The PGPR must have the ability to survive and multiply in rhizosphere microhabitats, in competition with native microbiota, at least for the time needed to express their beneficial plant growth promotion activities (Mártinez-Viveros et al., 2010). The processes involved in nutrient cycling by PGPR include nitrogen fixation, phosphate mobilization and the release of other nutrients to soil solution (Richardson et al., 2009; Barea and Richardson, 2015).

Beneficial plant mutualistic symbionts include the $\mathrm{N}_{2}$-fixing bacteria and the multifunctional arbuscular mycorrhizal (AM) fungi. Bacteria belonging to diverse genera, collectively termed as "rhizobia", are able to fix $\mathrm{N}_{2}$ in mutualistic symbiosis with legume plants (Olivares et al., 2013; de Bruijn, 2015). Other bacteria (actinomycetes), belonging to the genus Frankia, form $\mathrm{N}_{2}$-fixing nodules on the root of the so-called "actinorrhizal" plant species, having a great ecological importance (Normand et al., 2007). The other major group of mutualistic microbial symbionts are the AM fungi known to establish mycorrhizal associations with the roots of most plant species
(Smith and Read, 2008; van der Heijden et al., 2015). They belong to the phylum Glomeromycota (Schüßler et al., 2001).

AM formation can be considered as an adaptive strategy, which provides the plant with an increased ability for nutrient capture and cycling in soils with low nutrient availability. They are known to induce an increased tolerance to environmental stresses either biotic (pathogen attack) or abiotic (drought, salinity, heavy metals, organic pollutants), and to improve soil structure through the formation of aggregates necessary for a good soil tilth (Jeffries et al., 2003). Therefore, in sustainable agriculture the AM symbiosis plays a key role in helping the plant to be productive under adversity (Jeffries and Barea, 2012). Similarly, AM fungi play important roles in forest ecosystems (Borie et al., 2010).

AM-colonization changes the chemical composition of root exudates, while the AM soil mycelium itself introduces physical modifications into the environment surrounding the roots thereby affecting microbial structure and diversity. These processes give way to the so-called mycorrhizosphere, where specific microbial interactions occur (Barea et al., 2013a). Managing these interactions involving selected AM fungi and PGPR (mycorrhizosphere tailoring) is recognized as a feasible biotechnological tool in sustainable agriculture. Many co-inoculation experiments using selected AM fungi and rhizosphere microorganisms have been reported. These include interactions related to: (a) symbiotic $\mathrm{N}_{2}$-fixation; (b) phosphate mobilization; (c) phytoremediation of heavy metal contaminated soils; (d) biological control of root pathogens; and (e) improvement of soil quality (Barea et al., 2013a).

The scenarios for applying microbial technology include not only sustainable agriculture but also other ecosystemic issues. These refer to ecosystem restoration, recovering of endangered flora, enhancing resilience 
of plant communities, adaptive strategies for diversity conservation etc. (Barea et al., 2013b). In this chapter, the management of beneficial microbial activities is focused on sustainable agriculture. Essentially, there two major strategies for managing the soil microbiome, these are being based either on the development of microbial inoculants or on the manipulation of naturally existing microbial populations, including also non-culturable microorganisms.

\subsection{Implementing the technology for the production and application of high quality microbial inoculants}

According to J. Sanjuan (pers. comm.) for a successful application of microbial inoculants in agriculture we need to implement the following aspects: (a) to increase the scientific/technological bases of inoculum production and application; (b) to generate specific normative for each inoculant type and its application, either on the seeds or on the soil, or to the plant to be transplanted already microbized; (c) to establish quality control protocols; (d) to minimize the variability of the field results; and (e) to increase knowledge and dissemination by explicating advantages and limitations, and benefits for Society. Recently, Bashan et al. (2014) have published a comprehensive review on the formulation and practical perspectives of inoculant technology for PGPR. They recommend a number of top priorities of research to implement delivery systems for PGPR and rhizobia. Among others, these priorities include an indepth evaluation of carriers, an improvement survival of microorganisms in the inoculants, to enhance shelflife of the inoculants product, to use of multi-strain inoculants, to implement polymeric/encapsulated formulations, to follow low-cost technology, using local strains, to practice nursery inoculation for transplanted crops, etc. Several companies worldwide are producing PGPR inoculum products (Ravensberg, 2015; Kamilova et al., 2015).

Concerning production and application of AM fungal inoculants, the information has been reviewed recently (Jeffries and Barea, 2012; Singh et al., 2014). The main points addressed in these review articles can be summarized as follows. The difficulty in culturing the obligate symbionts AM fungi in the absence of their host plant is a major obstacle for massive inoculum production. Despite this problem, several companies worldwide are producing AM inoculum products, which are being applied in forestry, agriculture and horticulture. Specific procedures are required to multiply AM-fungi and to produce high quality inocula. The resulting materials (spores, hyphae, root fragments etc.), from "culturing" AM fungi, are added to different carriers, resulting in a wide range of formulations, including encapsulation, to be applied at an agronomical scale using different techniques. Recent developments in AM-inoculum production systems include the in vitro monoxenic root organ cultures. Inoculation of seedlings (nursery production) is potentially a good method for establishing selected fungi in the roots before potting on or planting-out into the field, as is the case with horticulture and plantation crops, including fruit farming.

Apart from microbial inoculation, there are other challenging opportunities to exploit the beneficial activities of soil microorganisms. The perspectives for the successful manipulation of naturally existing microbial population, towards a sustainable production of healthy foods, are becoming feasible thank to recent advances in the new system-based strategies to study plant-microbiome interactions. Particularly, understanding of these interactions is being facilitated by the already available, cultureindependent, molecular techniques, which are discussed in the next section. 


\section{Molecular ecology approaches for a better understanding of root-microbiome interactions}

The lack of appropriate methodologies have constrained advances for a comprehensive understanding of the mechanisms underlying plant-microbe interactions in the rhizosphere, Difficulties rely mainly on the need of profiling a great array of processes where the large and diverse microbial communities, predominantly constituted by unculturable microorganisms, are involved (Carvalhais et al., 2013). However, a plethora of culture-independent molecular techniques are becoming available, and are currently being applied either to decipher the hidden diversity of microorganisms inhabiting soil and rhizosphere microenvironments, or to dissect the molecular bases of the plant-microbiome interactions, as summarized in Figure 1. These techniques, based on molecular approaches, are also fundamental to evaluate the impacts of perturbations provoked by biotic and abiotic stress factors on soil microbiome diversity and on plant-microbe interactions, in the current scenario of global change.

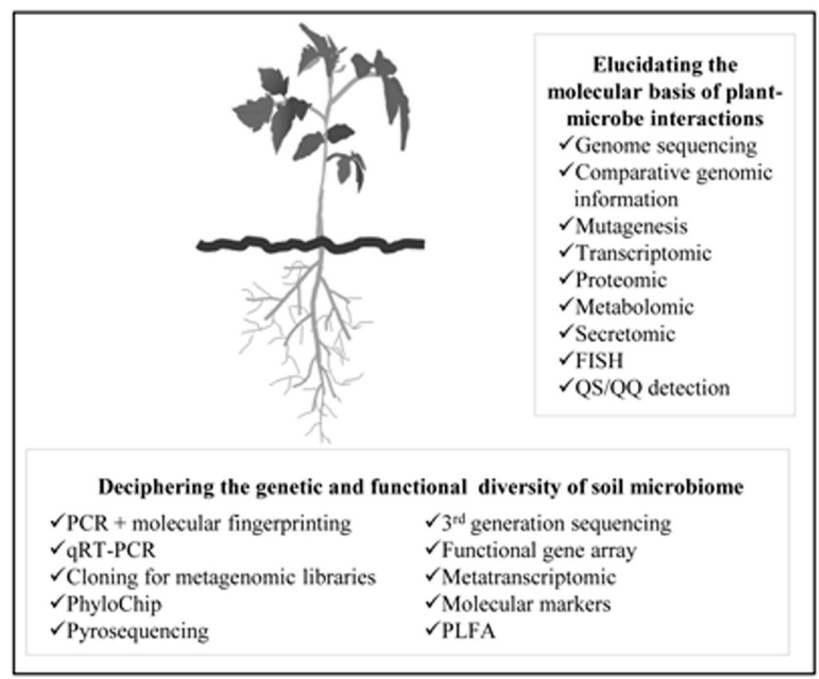

Figure 1. Culture-independent (system-based) molecular techniques currently used to decipher the diversity of microorganisms inhabiting soil and rhizosphere microenvironments, or to dissect the molecular bases of the plant-microbiome interactions. Ideas based on Barret et al. (2013); Chauhan et al. (2013).

A great advance in molecular ecology technique for analyzing soil microbial diversity relies on that nowadays is not necessary to isolate the microorganisms (those culturable). In fact, the whole soil microbiome, including the unculturable components, can be lysed directly in soil to further extract their DNA/RNA and other biochemical markers. As it is well known, the DNA characterizes the phylogenetic identity and functional capability of the microbes, while RNA refers the genes, which are expressed in a given situation. As an example of biochemical markers, phospholipid fatty acids 
(PLFAs) extracted from cell membranes are used as indicators of soil microbial community structure (Hirsch et al., 2013a).

The culture-independent techniques currently used to analyze the genetic and functional diversity of microbial communities in the bulk of soil and in the rhizosphere have been recently reviewed (Barret et al., 2013; Chauhan et al., 2013; Hirsch et al., 2013a; Schreiter et al., 2015). Key information from these comprehensive and extended chapters is briefly and pragmatically summarized below. The start point is the processes for extracting DNA/RNA from soil samples. The nucleic acids can be isotopically labeled prior to their extraction from the environmental samples for further monitoring. The collective genome of microbes, i. e. the DNA isolated from microbial communities, constitutes an entity termed as a metagenome. Hence, metagenomics refers to the isolation and cloning of large intact DNA fragments, which included several genes and operons. The total DNA extracted from environmental samples can be submitted to different techniques based on cloning approaches, PCR amplification, high throughput sequencing, or microarray hybridization. Cloning based approaches are allowing for the construction of metagenomic libraries that can be screened either for structural and functional genes or for phenotypic traits related to proteins, including enzymes, and secondary metabolites profiling. Bioinformatic based approaches are always involved in soil microbial metagenomics studies.

The application of the PCR technique, and its derived quantitative approach (qPCR), to microbial DNA extracted from soil communities have resulted in a major break-through for deciphering microbial diversity. The small ribosomal subunit sequences (16S for bacteria and $18 \mathrm{~S}$ for fungi) are target molecular markers of microbial communities. Particularly, the comparative gene analysis of $16 \mathrm{~S} / 18 \mathrm{~S}$ ribosomal
RNA (rRNA) is the most frequent molecular method used for microbial identification. Actually, rRNA gene is a universal marker as encoded in nearly all microbial genomes. The more highly conserved regions in the rRNA gene sequence can be used to construct "universal" primers to amplify this gene from the DNA extracted from environmental samples. The sequence analysis of cloned $16 \mathrm{~S} / 18 \mathrm{~S}$ rRNA genes is the basis to compare, composition, richness, evenness, and structure of microbial communities. The PCR products (amplicons) sharing similar or identical variable region are considered as operational taxonomic units (OTUs).

Assessing the diversity of PCR products can be performed by well-known molecular typing methods, which allow for a molecular fingerprint for the structure of a target microbial community. The amplicon diversity can also be assessed by other methods involving cloning and sequencing. These approaches are being nowadays facilitated by the high throughput next-generation sequencing methods able to assess directly the sequence of the $16 \mathrm{~S} / 18 \mathrm{~S}$ rDNA amplicons. Moreover, the $3^{\text {rd }}$ generation sequencing technology, SMRT ("single molecule real time sequencing”), does not need a previous PCR process as is based on a single DNA molecule. Alternatively, to the fingerprinting strategies other approaches are being used to monitor the abundance of specific taxonomic group in the communities such as the functional gene microarray-based GeoChip and PhytoChip methods. Other high throughput sequencing techniques, such as the pyrosequencing of $16 \mathrm{~S} / 18 \mathrm{~S}$ rDNA amplicons, is being used to monitor the taxonomic identity (from the phylum to the genus level) of microbial communities in different soils and biomes.

The functional diversity of the microbial community can be assessed by amplifying specific functional genes (functional markers) responsible for specific 
metabolic processes. Since in functional diversity studies some insights on microbial activity are needed, strategies based on transcript abundance have been employed as monitored by qRT-PCR. Functional gene arrays have been developed to evaluate the expression of transcripts from different genes and this has been employed to assess the activity of specific functional microbial activities. In this context, shotgun sequencing involving DNA microarrays containing environmental cDNA has been used to compare the response of soil microbiome to external impacts at a transcriptional level. Metatranscriptome analysis of RNA from environmental samples is being used to compare the effect of environmental factors on the transcriptomes of microbial communities.

Diverse approaches are currently used to understand the molecular basis of interactions among plants and microbial communities in the rhizosphere. A basic concept is that plant specific rhizodeposition (carboncontaining materials of plant origin), including root exudation, drives the selection of microbial diversity that the target plant recruit in its rhizosphere (Hirsch et al., 2013b). Since the root-associated microorganisms, stimulated by rhizodeposition, carry out specific activities impacting on plant nutrition and health, a feedback loop between plant and microorganisms is generated (Zancarini et al., 2013). These authors point out that plant functioning, as affected by the activities of microbial communities, can be analyzed thanks to high throughput plant phenotyping, while the effects of plant genotype on the diversity and functioning of microbial communities can be analyzed thanks to molecular ecology tools (sequencing, meta- "omics", etc.), as illustrated in Figure 2.

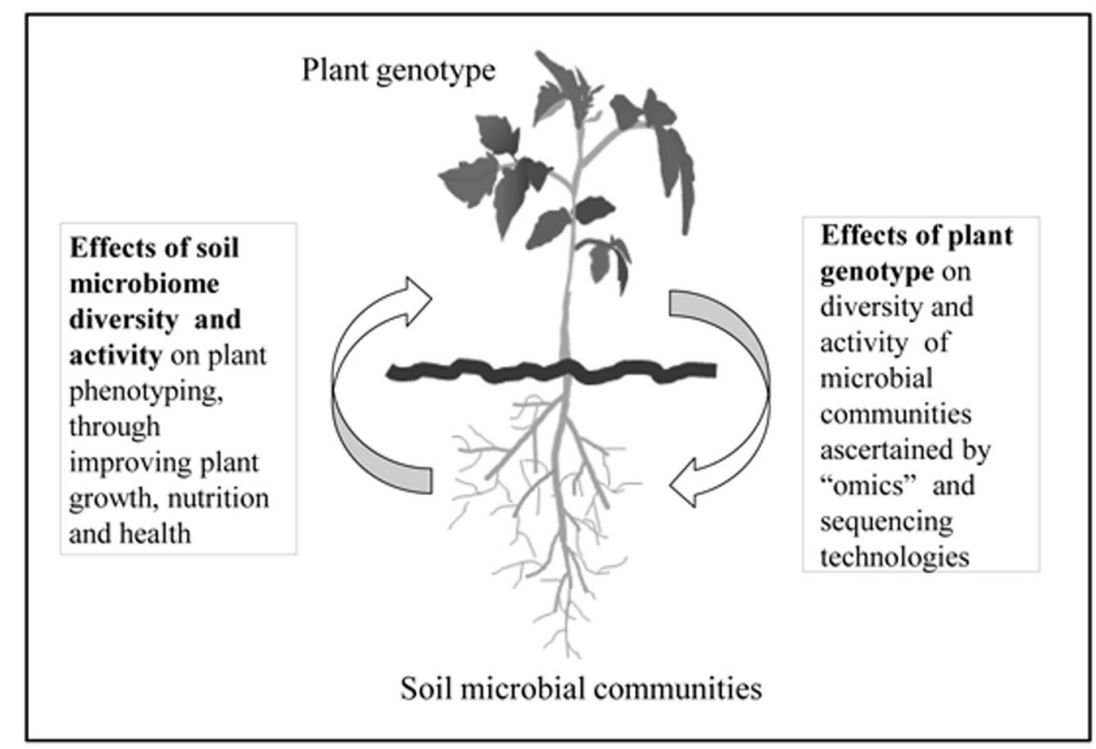

Figure 2. Feedback activities during plant-microbe interactions in the rhizosphere. Ideas based on Zankarini et al. (2013) 
Application of novel technologies has allow for detection and functioning of signal molecules and signaling processes in the trans-kingdom communications leading to the establishment of PGPR-plant assemblages. Drogue et al. (2013) divided these signal molecules according to whether they are produced by: (i) the plant, which affects gene expression by PGPR, (ii) the PGPR, that affect plant nutrition and health, and (iii) PGPR, which affect gene expression by other PGPR. The primary metabolites of plant origin include amino acids, organic acids, vitamins, proteins, sugars, etc., while the secondary ones include flavonoids, phenol, phytohormones, etc. The small signal molecules produced by PGPR able to affect directly plant growth and or stress alleviation include the different classic phytohormones, acyl homoserine lactones (AHLs) and diacetylphloroglucinol (DAPG). These molecules are involved in the control of root architecture, phytostimulation, induction of systemic resistance (ISR), stimulation of root exudation, etc. (OrtizCastro and López-Bucio, 2013; Drogue et al., 2013). Interestingly, signaling processes in plant-microbe symbioses related to nutrient supply, i. e. nodulation and mycorrhization follow similar pathways, as also occurs in plant-pathogen interactions (Jayaraman et al., 2012; Bonfante and Desirò, 2015).

System-based strategies addressed to decipher the molecular basis of plant microbiome interactions at the genomic, transcriptomic, proteomic and metabolomic levels, based on functional genomics analysis of model soil microbes, have recently been reviewed (Barret $e t$ al., 2013). Consequently, only a brief summary of this information is given below. Some approaches are DNA-based and one of them relies on sequencing the genome. Bioinformatic studies of different bacterial genomes are used to establish the distribution and abundance of functional traits within the genomes, for example, the traits involved in rhizosphere colonization. Other approaches are RNA-based and rely on monitoring bacterial gene expression by either detecting active promoter or evaluating the abundance of the mRNAs. Actually, the relative abundance of mRNAs in contrasting situations (comparative transcriptome) can be evaluated by differential gene expression studies based on microarrays. Carvalhais et al. (2013) proposed rhizosphere metatranscriptomics as a challenging approach to provide microbial activity profiles by assessing the expressed functional genes responsible for key rhizosphere interactions.

Another aspect addressed in the review chapter of Barret et al., (2013) is the identification and prospection of the spatiotemporal production of signal molecules in the rhizosphere affecting microbial communities. Among these molecules are fundamental those involved in the microbial quorum sensing (QS) and the plant-derived quorum quenching (QQ). The QS system is also known to modulate biofilm formation and the production of antibiotic, siderophores and secondary metabolites. Microbial QS molecules, like AHLs, seem to be involved also in the induction of systemic resistance against leaf pathogens.

New analytical techniques are currently being proposed to help understanding the dynamics of rhizosphere colonization where many interactions are taken place (Barret et al., 2013). One of the most commonly used techniques is the fluorescence in situ hybridization (FISH), where DNA/RNA probes label microbes containing homologous sequences and enable localization of individuals. Variants of the FISH like the confocal laser scanning microscopy (CLSM)FISH, among others, are improving the sensitivity of the technique. A new alternative approach, termed secondary ion mass spectrometry (SIMS) is enabling the analysis of the structure of microbial communities. Another tool to follow microbial functioning dynamics in the rhizosphere is that based on the characterization of nutrient availability. These include the use of stable 
isotope labeling or biosensors. Using stable isotopes (nutrients) in combination with FISHs approaches and oligonucleotide probes makes possible to identify the microbial or plant cells, which have taken up the labeled nutrient.
The analysis of the genome of the AM fungi (GianinazziPearson et al., 2012) and AM functioning, when symbiotically associated to plants (Franken et al., 2012; Bonfante and Desiró, 2015), has demanded particular research approaches based on using molecular tools.

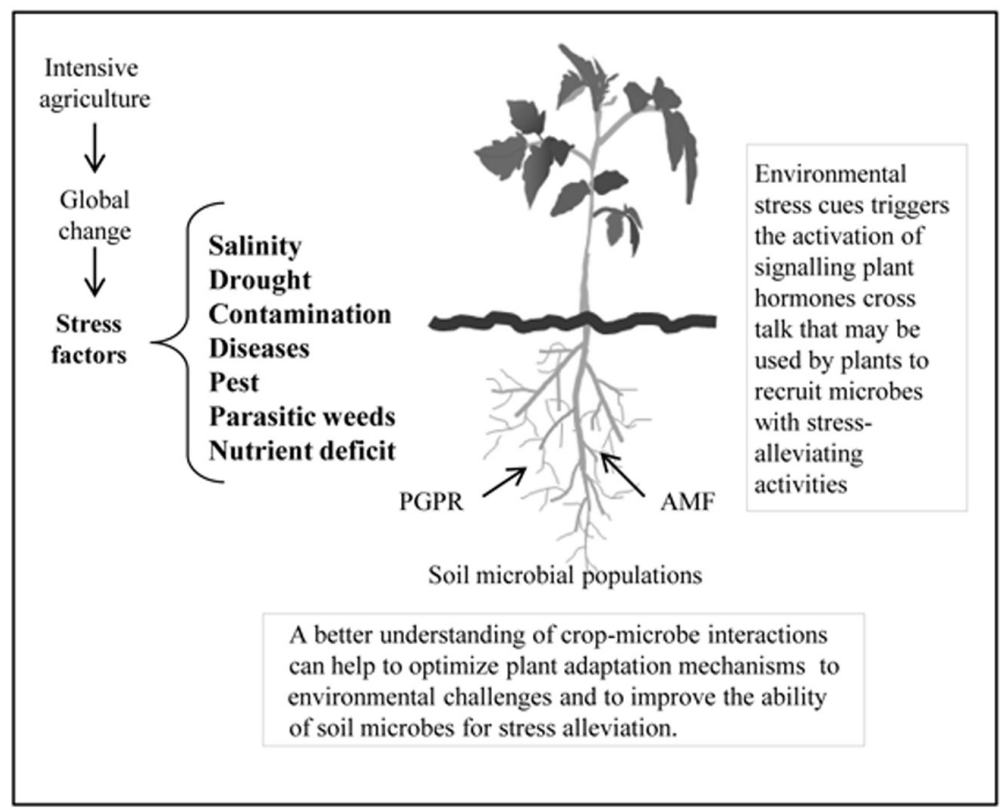

Figure 3. The impact of environmental stress challenges on plant-microbe interactions. Ideas based on Zolla et al. (2013).

\section{Improving the ability of soil microbes for stress alleviation in crops based on a better understanding of plant-microbiome interactions}

Diverse types of stress factors, including salinity, drought, nutrient deficits, contamination, diseases and pests, etc. can alter plant-microbe interactions in the rhizosphere (Figure 3). Recent research is evidencing that plant perception of environmental stress cues triggers the activation of signaling molecules, phytohormones play a key role. This signal input is followed by a signal processing, and finally by a signal output, which enables plants to respond to these environmental constraints. As plants are exposed to multiple stresses simultaneously, appropriate metaanalyses reveal a complex regulation of plant growth and immunity. Understanding how phytohormones interact in the signaling network is fundamental to learn how plant-microbiome systems thrive and survive in stressed environments. This understanding is relevant to design biotechnological strategies to optimize plant adaptation mechanisms and to improve the ability of soil microbes for stress alleviation in crops (Pozo et al., 2015). Mechanisms involved in 
plant-microbe interactions under stress situations are poorly understood. However, ongoing research is evidencing the involvement of changes in plant morphology, physiology, transporter activity and root exudation profiles, changes that can induce the plant to recruit microbes with stress-alleviating capacities, a strategy able to help crop productivity under stress (Zolla et al., 2013).

As stress factors cause detrimental impacts on the functionality/productivity of agricultural systems, the role of rhizosphere microorganisms in helping plants to thrive in adverse conditions has recently been discussed (Barea et al., 2013b) The aim of this section is to analyze how we can improve the ability of soil microorganisms for stress alleviation in crops, as aided by a better understanding of plant-microbe interaction based on the already available meta "omic" and sequencing approaches.

\subsection{Improving the ability of soil microorganisms for the biocontrol of pathogens (diseases, pests...)}

Rhizospheric and root endophytic microbes, including PGPR, Trichoderma spp. and AM fungi, protect plants against pathogens by competition for space and nutrients, antibiosis (for PGPR and Trichoderma), mycoparasitism (for Trichoderma) and by inducing plant defense mechanisms (Barea et al., 2013a). Defense priming is the preconditioning of immunity induced by microbial colonization, fundamental for an efficient protection against pathogens. Priming also acts systemically on distant parts of the root and shoots thereby inducing systemic resistance (ISR) to protect efficiently plants against both roots and foliar pathogens (Selosse et al., 2014).

The AM symbiosis protects plants against deleterious organisms including microbial pathogens, herbivorous insects and parasitic plants. AM colonization can prime plant immunity by boosting the plant ability to respond to pathogen attack, where jasmonic acid (JA) plays a key role. ISR in AM symbiosis is termed Mycorrhiza Induced Resistance (MIR). The identification of defense regulatory elements coordinating AM development and MIR is a major challenge for research since can facilitate the development of biotechnological strategies for improving the use of AM fungi in the integrated management of pests and diseases (Pozo and Azcón-Aguilar, 2007; Jung et al., 2012; Pozo et al., 2013). In addition, PGPR, Trichoderma spp, and non-pathogenic Fusarium strains also prime local resistance and ISR, as they produce microbe-associated molecular patterns (MAMPs), which trigger immune responses. The priming effects of MAMPs rely on that they activate the JA signaling pathway, which regulates the inducible plant defenses (Pozo et al., 2015). Indeed, in the plant hormone signaling crosstalk, which regulates plant defense and development in microbe-plantinsect interactions, JA results in the main hormone at switching from growth to defense responses (Pangesti et al., 2013).

Because the effectiveness of biocontrol practices is affected by the prevailing environmental conditions, biocontrol-related research has to envisage the challenge of finding appropriate screening procedures to select microorganisms able to be highly effective under the current changing scenarios. Understanding the impact of the environment on the biocontrol agent performance will help to predict the resulting output and to develop effective combinations of antagonistic microorganisms. A major challenge in rhizosphere biotechnology is to exploit the prophylactic ability of AM fungi in association with antagonist microorganisms. The final aim is to find out an enhanced capacity for bioprotection achieved by the combination of the mechanisms used by each organism individually. While experimental evidence 
supports that mycorrhizosphere management is a promising biotechnological tool to enhance plant resistance/tolerance to pathogen attack, research on the optimal microbial combinations is essential for the successful application in sustainable agriculture (Barea et al., 2013a).

Recent research on agricultural weed control is revealing strategies focused on the initial steps in the host-parasite interaction. Actually, parasitic weeds are difficult to control because most of their life cycle occurs underground. The strigolactones, a new class of plant hormones are signaling molecules, which stimulate germination of root parasitic plant seeds. Besides, it has been shown that strigolactones are involved in root colonization by the AM fungi. Upon AM colonization, plants reduce the production of strigolactones thereby lowering parasitic plant infection, and consequently diminishing the deleterious effect of these weeds on plant fitness and yield (López-Ráez et al., 2012). The possible applicability of the AM symbiosis in weed control, based on AM activities regulating plant production of strigolactones, as an agricultural practice in the context of sustainability issues, has been discussed recently (Jung et al., 2012; Pozo et al., 2013).

\subsection{Improving the ability of soil microorganisms for} alleviating the negative effects of osmotic stressors (drought, salinity...)

The level of aridity in many land areas of the world has increased progressively rising thereby drought and salinity problems. To cope with such osmotic stressors plants must develop a number of adaptation mechanisms including mainly a fine regulation of their water uptake capacity and transpiration rates, and the activation of the antioxidant machinery to overcome the overproduction of reactive oxygen species (ROS) caused by the stress. The related available information has been discussed recently (Dimkpa et al., 2009; Aroca et al., 2012; Dood and Pérez-Alfocea, 2012; Groppa et al., 2012; Porcel et al. 2012; Ruiz-Lozano et al., 2012a; b; Bal et al., 2013; Calvo-Polanco et al., 2013; Barzana et al., 2014), and is briefly summarized here.

The two general mechanisms (maintaining water and ROS balance) may be ameliorated by both the establishment of the AM symbiosis and by inoculation with PGPR, which act through diverse specific mechanisms. These can be summarized as follows: (i) cell osmoregulation (related to the accumulation of the compatible solutes such as proline, glycine, betaine, soluble sugars, pinitol and mannitol); (ii) ionic homeostasis (based on ion balance and compartmentalization and related to maintaining a fine balance of $\mathrm{K}^{+}: \mathrm{Na}^{+}$and $\mathrm{Ca}^{2+}: \mathrm{Na}^{+}$ratios); (iii) regulation of root water uptake and redistribution along plant tissues by aquaporins (where a phytohormone crosstalk is involved); (iv) antioxidant defense (to compensate the production of harmful reactive oxygen species (ROS); and (v) maintenance of photosynthetic capacity. Such microbial activities result in a better regulation of plant water status and contribute to increase plant resistance to osmotic stress conditions. Finally, the improved water uptake capacity of microbiologically inoculated plants allows them to have higher transpiration rates and hence higher photosynthetic rates under conditions of water deficit.

Particular attention is receiving the role played by AM fungi and other rhizosphere microorganisms to improve plant water status based on the improvement of root hydraulic conductance, which ultimately depends on aquaporin functioning (Aroca et al., 2012; Groppa et al., 2012; Ruiz-Lozano et al., 2012a; b; Calvo-Polanco et al., 2013; Barzana et al., 2014). Aquaporins are membrane intrinsic proteins that allow for water and other small neutral molecules 
to pass across biological membranes following an osmotic gradient (Chaumont and Tyerman, 2014; Li et al., 2014). The improvement of the nutritional status of microbial-inoculated plants, together with the release of volatile organic compounds (VOCs) by some microorganisms, regulates root aquaporins expression and/or activity, and thereby root hydraulic conductance. The regulation of root aquaporins is based on phytohorme interactions, in which plant levels of abscisic acid (ABA) appear to play a central role (Dood and Pérez-Alfocea, 2012; Groppa et al., 2012; Barzana et al., 2014).

While is becoming clear that AM fungi and other rhizosphere microorganisms are able to increase resistance/tolerance to osmotic stressors, further studies are still needed to yield a comprehensive analysis of the transfer of this knowledge to natural ecology. This is fundamental because soil and rhizosphere microorganisms are key factors for plant survival under a changing environment where plants are going to be exposed to adversity on the oncoming years, as driven by the climatic change (Duarte et al., 2006).

\subsection{Improving the ability of soil microorganisms for the phytoremediation of contaminated soil}

Plant-associated microorganisms, i.e. AM fungi and bacteria can enhance plant abilities for the remediation (phytoremediation) of environments contaminated with heavy metals, radionuclides or organic xenobiotics (including volatile organic compounds, oil derived alkanes or polycyclic aromatic hydrocarbons). Current phytoremediation technologies use plants and their associated microorganisms to remove, transfer, stabilize, decrease, and/or decompose pollutants in the environment (Azcón et al., 2013). To be successful for phytoremediation purposes plants must be capable to thrive in polluted environments while their associated microorganisms have also to be adapted to the presence of contaminants (Pongrac et al., 2013). Most phytoremediation research involving plantassociated microorganisms concerns heavy metals (HMs) cleaning or organic xenobiotic degradation (Azcón et al., 2013).

The mechanisms underlying the role of plant associated bacteria in phytoremediation of environments contaminated with HMs or organic xenobiotics, in general (Germaine et al., 2013), and alkanes, in particular (Afzal et al., 2013), have recently been analyzed. These mechanisms include improvement of plant growth, nutrient (P and $\mathrm{N}$ ) supply, production of Fe-binding siderophores, plant hormones production, enhanced ACC-deaminase activity (ethylene reductions), organic xenobiotic degradation, etc. Another mechanism for improving phytoremediation is the bio-augmentation of plant associated microbial communities based on horizontal gene transfer (Germaine et al., 2013). This mechanism, a challenge of future research, relies on that many resistance genes involved in HMs bioremediation processes are located in plasmids that can be transferred within the bacterial communities. The complete genomes of a number of plant-associated bacteria are becoming available. This, together with the genome sequence of diverse plant host, would facilitate establishing the molecular communications between plant and bacteria, a key step to provide new insights allowing for design improved strategies in phytoremediation (Germaine et al., 2013; Afzal et al., 2013).

The AM fungi have also evolved a series of strategies to restrict entry of toxic metal species and to keep intracellular metal homeostasis (Ferrol et al., 2009; González-Guerrero et al. 2009; 2010). According to these authors, the mechanisms of HM tolerance in AM fungi include reduction of metal uptake and/ or increased efflux, metal immobilization, e.g. cellwall adsorption, extracellular metal sequestration, 
intracellular chelation by e.g. metallothioneins or phytochelatins, and metal compartmentalization into spores and vesicles. Specific metal transporters regulate cytosolic metal ion concentrations and translate the excess of metal within vacuoles, where they would cause less damage. AM fungi have also evolved mechanisms to fight against the oxidative stress produced by HMs or to repair the oxidative damage. Increased HM tolerance of mycorrhizal plants may be related to extensive changes in gene expression and protein synthesis induced by the symbiosis itself. Glomalin-related soil proteins produced by AM fungi can irreversibly sequester HMs (Cornejo et al., 2008), thereby contributing to metal stabilization in the soil.

Understanding the key molecular determinants of metal homeostasis in AM fungi is challenging. To get some insights into the underlying mechanisms, a genome-wide analysis of HMs transporters was undertaken (Tamayo et al., 2014), making use of the recently published whole genome of the AM fungus Rhizophagus irregularis. This in silico analysis allowed identification of 30 open reading frames in the $R$. irregularis genome, which potentially encode HMs transporters. The authors depict a comprehensive scheme of the mechanisms involved. A current challenge is to characterize the functionally of these transporters and to identify their location and roles in the AM symbiosis.

Interactions between HM-adapted rhizobacteria and AM fungi have been investigated in diverse experiments (Medina and Azcón, 2010). The main achievements resulting from these experiments were: (i) the target bacteria accumulated large amounts of HMs; (ii) co-inoculation enhanced plant establishment and growth, and lowered HM concentrations in plants, supporting a phytostabilization-based activity, while the total HM content in plant shoots was higher in dually-inoculated plants, suggesting a phytoextraction activity; and (iii) inoculated HM-adapted bacteria increased enzymatic activities and plant hormone production in the mycorrhizosphere. Inoculation of autochthonous AM fungi and PGPR, together with the application of treated agrowaste residue, changed the bacterial community structure and enhanced phytoextraction to remediate HM contaminated soils. A challenging topic for future research is to realize the phytoremediation effects of mycorrhizosphere interactions under field conditions.

\section{Engeneering the rhizosphere to encourage beneficial microbe establishment: a great challenge for the future}

Diverse research approaches are currently addressed trying to ascertain whether the rhizosphere can be engineered to encourage beneficial organisms, while prevent presence of pathogens. The related research topics offer many challenges because there are many gaps in our understanding on the ad hoc research strategies. Undoubtedly, getting biased rhizosphere opens new opportunities for future agricultural developments based in exploiting the beneficial microbial services to reduce the inputs of agrochemicals thereby reaching sustainable environmental and economical goals.

\subsection{Learning how plants shape microbial community structure in the rhizosphere}

Current research is realizing that plants can structure their root-associated microbial communities, concerning both diversity and functions (Achouak and Haichar, 2013; Hirsch et al., 2013b). Particularly, Achouak and Haichar (2013) used the stable isotope probing (SIP) together with fingerprinting approaches as a molecular detection tool to analyze the impact of the plant species on their rhizosphere microbiome. 
They confirmed the differential impact of each target plant species on the genetic and functional diversity of the plant-associated bacterial communities. Therefore, such ability of the plants for shaping microbial communities in their rhizospheres appears as a new opportunity for linking structure and function of the root-microbiome related to nutrient supply and plant protection. Carbon compounds and signal molecules from root exudates are the main drivers of plant specific effects on rhizosphere bacteria and their proteomes. Actually, the identity and quality of rhizodeposits varies from plant to plant thereby attracting a specific set of bacteria to the rhizosphere and providing them with a selective pressure to stimulate bacteria to compete and persist (Hirsch et al., 2013b), a property which is depending on plant age (Spence and Bais, 2013).

Harnessing the rhizosphere microbial communities through agricultural managements

In a comprehensive analysis of the available experimental evidence, combined with theoretical models, Bakker et al. (2012) outlined strategies to manipulate root exudation for a plant-driven selection of beneficial rhizosphere microbes. The main ideas/conclusions of this review article are summarized here. In some cases, the ideas are based on speculations but these have a reasonable feasibility in the nearest future.

An example of harnessing the rhizosphere microbiome derives from the existence of plant-microbe coadaptation, involving a shared evolution history of interactions between plants and soil microbiome. In a co-adapted rhizosphere, pathogens can be present but their activities are controlled. In contrast, when an agricultural plant species is moved to other parts of the world, as happens with the current agricultural global exchange, the plant will grow in association with a microbiome, which has not shared evolutionary history. The target crop will associate with an un- adapted microbiome, which does not constrain pathogen establishment and therefore becoming susceptible to diseases.

According to Bakker et al. (2012), there are two main strategies for manipulating the plant to recruit beneficial microorganisms in its rhizosphere, both of them are based on plant breeding, and are addressed to foster beneficial microbial services for improving agricultural developments. One of these alternate paths relies on develop plants able to shape their microbiome by targeting particular taxa for specific functions i. e. $\mathrm{N}_{2}$-fixation, P-mobilization, biocontrol, etc. The other approach is based on develop plants able to shape their microbiome for broad characteristics related to promotion of plant growth and health. All in all, in the nearest future it appears that the more feasible approach to enhance beneficial microbial services in agriculture is the direct manipulation of the soil microbiome. Particularly, a target aim is to reconstruct a minimal rhizosphere microbiome able to provide a maximized benefit to plant at a minimal photosynthetic cost (Raaijmakers, 2015).

A challenging strategy which offers opportunities to enable plants to recruit microorganisms targeted for specific functions, is that aimed at engineering nitrogen-fixing cereals (Rogers and Oldroyd, 2014; Oldroyd and Dixon, 2014; Venkateshwaran, 2015).

\subsection{The "biased rhizosphere" concept/action}

The "biased rhizosphere" concept is based on the possibility of provoking the production by the plant of special compounds that can be catabolized only by target beneficial bacteria introduced as inoculants, e.g. PGPR (Savka et al., 2013). These authors have revised the biased rhizosphere concept and provide pioneering insights on its origin and significance. The origin of the biased rhizosphere concept derived from an analysis of the interactions between rhizobia and plant (generated 
by rhizopine-like molecules) and Agrobacterium and plant (generated by opine-like molecules), specific compounds able to foster such interactions. Savka et al. (2013) also discuss how the throughput sequencing methods have provided comparative genomic information on plant-associated bacteria while the omics technologies have facilitated further research of these interactions. For example, the time-course effect of host rhizosphere chemistry can be monitored in studying microbial community structure and metagenomics. Future studies have to be undertaken to find specific metabolite-plant species-microbe combinations. Deciphering the biotic and abiotic plant factors that shape the plant-associated microbiome through biasing the rhizosphere offers many challenges that current research is trying to envisage. According to Savka et al. (2013) future work on plants must focus on reprogramming transport functions, while those on microorganisms have to focus on the uptake secreted nutrients and the time-course changes in the microbial community structure. A combination of all of these approaches can improve our understanding on how to enhance the competitiveness and persistence of bacteria in the biased rhizosphere to finally improve plant health and agro-ecosystem productivity.

\section{Concluding remarks}

Exploiting the interactions between soil microbial communities and crops is a relevant approach to increase food production for the growing world population at the lowest environmental costs, in the current scenario of global change. Essentially, there are two major strategies for managing the soil microbiome, these are being based either on the development of microbial inoculants or on the manipulation of naturally existing microbial populations. Both rhizosphere bacteria (PGPR) and fungi, either saprophytic or endophytic symbionts (with special reference to $\mathrm{N}_{2}$-fixing rhizobia and AM fungi) are protagonists of applied microbial biotechnology in agriculture. Particular emphasis is being paid to formulation, quality control and modes of application of microbial inoculants. Many of the mechanisms underlying plant-microbe interactions in the rhizosphere are still poorly understood. Difficulties rely mainly on the need of profiling the great array of processes in which are involved the large and diverse microbial communities, predominantly constituted by unculturable microorganisms. A plethora of cultureindependent molecular techniques is becoming available, and is currently being applied either to decipher the hidden diversity of microorganisms inhabiting soil and rhizosphere microenvironments, or to define the molecular bases of the plant-microbiome interactions. Diverse types of stress factors, including salinity, drought, nutrient deficits, contamination, diseases and pests, cause detrimental impacts on the functionality/productivity of agricultural systems. A signaling network orchestrated plant-microbiome interactions needed to thrive and survive in stressed environments. Understanding this signal crosstalk is fundamental to design biotechnological strategies to optimize plant adaptation mechanisms and to improve the ability of soil microbes for stress alleviation in crops. Several approaches are currently addressed to ascertain whether the rhizosphere can be engineered (biased) to encourage beneficial organisms, while prevent presence of pathogens. The target research topics offer many challenges because there are many gaps in our understanding. As a general conclusion, we can say that many achievements have been reached with the application of microbial biotechnology in agriculture but many challenges as well as opportunities need to be explored for the future sustainable agricultural developments. 


\section{Acknowledgements and Dedicatory}

The author of this review chapter thanks the Spanish National Research Programs AGL201239057-C02-02 project and to the PAIDI Program P11CVI-7640 project for financial support.

This chapter is dedicated to Prof. José Olivares Pascual who, during more than half a century (19592015), carried out and coordinated, and still continues advising on, scientific research of excellence in the Departamento de Microbiología del Suelo, Estación Experimental del Zaidín, CSIC, Granada, Spain. The author also thanks Prof. Olivares for its critical reading of this manuscript.

\section{References}

Achouak, W., Haichar, F.Z. 2013. Shaping of microbial community structure and function in the rhizosphere by four diverse plant species. In: F.J. de Bruijn (ed). Molecular Microbial Ecology of the Rhizosphere, vol 1. Wiley Blackwell, Hoboken, New Jersey, USA, pp: 161-167.

Afzal, M., Yousaf, S., Reichenauer, T.G., Sessitsch, A. 2013. Ecology of alkane-degrading bacteria and their interaction with the plant. In: F.J. de Bruijn (ed). Molecular Microbial Ecology of the Rhizosphere, vol 2. Wiley Blackwell, Hoboken, New Jersey, USA, pp: 975-989.

Altieri, M.A. 2004. Linking ecologists and traditional farmers in the search for sustainable agriculture. Front. Ecol. Environ. 2, 35-42.

Aroca, R., Porcel, R., Ruíz-Lozano, J.M. 2012. Regulation of root water uptake under abiotic stress conditions. J. Exp. Bot. 63, 43-57.

Azcón, R., Medina, A., Aroca, R., Ruíz-Lozano, J.M. 2013. Abiotic stress remediation by the arbuscular mycorrhizal symbiosis and rhizosphere bacteria/ yeast interactions. In: F.J. de Bruijn (ed).
Molecular Microbial Ecology of the Rhizosphere. Wiley Blackwell, Hoboken, New Jersey, USA, pp: 991-1002.

Bakker, M.G., Manter, D.K., Sheflin, A.M., Weir, T.L., Vivanco, J.M. 2012. Harnessing the rhizosphere microbiome through plant breeding and agricultural management. Plant Soil 360, $1-13$.

Bal, H.B., Nayak, L., Das, S., Adhya, T.K. 2013. Isolation of ACC deaminase producing PGPR from rice rhizosphere and evaluating their plant growth promoting activity under salt stress. Plant Soil 366, 93-105.

Barea, J.M., Pozo, M.J., Azcón, R., AzcónAguilar, C. 2013a. Microbial interactions in the rhizosphere. In: F.J. de Bruijn (ed). Molecular Microbial Ecology of the Rhizosphere, vol 1. Wiley Blackwell, Hoboken, New Jersey, USA, pp: 29-44.

Barea, J.M., Pozo, M.J., López-Ráez, J.A., Aroca, R., Ruíz-Lozano, J.M., Ferrol, N., Azcón, R., AzcónAguilar, C. 2013b. Arbuscular Mycorrhizas and their significance in promoting soil-plant systems sustainability against environmental stresses In: B. Rodelas, J. González-López. (eds). Beneficial Plant-Microbial Interactions: Ecology and Applications. USA, CRC Press, pp: 353-387.

Barea, J.M., Richardson, A.E. 2015. Phosphate mobilisation by soil microorganisms. In: B. Lugtenberg (ed). Principles of Plant-Microbe Interactions. Springer International Publishing Switzerland, Heidelberg, pp: 225-234.

Barret, M., Tan, H., Egan, F., Morrissey, J.P., Reen, J., O'Gara, F. 2013. Exploiting new systems-based strategies to elucidate plant-bacterial interactions in the rhizosphere. In: F.J. de Bruijn (ed). Molecular Microbial Ecology of the Rhizosphere, vol 1. Wiley Blackwell, Hoboken, New Jersey, USA, pp: 57-68. 
Barzana, G., Aroca, R., Bienert, G.P., Chaumont, F., Manuel Ruiz-Lozano, J. 2014. New insights into the regulation of aquaporins by the arbuscular mycorrhizal symbiosis in maize plants under drought stress and possible implications for plant performance. Mol. Plant-Microbe Interact. 27, 349-363.

Bashan, Y., de-Bashan, L.E., Prabhu, S.R., Hernandez, J.-P. 2014. Advances in plant growth-promoting bacterial inoculant technology: formulations and practical perspectives (1998-2013). Plant Soil 378, 1-33.

Bonfante, P., Desirò, A. 2015. Arbuscular mycorrhizas: the lives of beneficial fungi and their plant host. In: B. Lugtenberg (ed). Principles of Plant-Microbe Interactions. Springer International Publishing Switzerland, Heidelberg, pp: 235-245.

Borie, F., Rubio, R., Morales, A., Curaqueo, G., Cornejo, P. 2010. Arbuscular mycorrhizae in agricultural and forest ecosystems in Chile. J. Soil Sci. Plant Nutr. 10, 185-206.

Brader, G., Compant, S., Mitter, B., Trognitz, F., Sessitsch, A. 2014. Metabolic potential of endophytic bacteria. Curr. Opin. Biotechnol. 27, 30-37.

Browne, P., Barret, M., Morrissey, J.P., O’Gara, F. 2013. Molecular-based strategies to exploit the inorganic phosphate-solubilization ability of Pseudomonas in Sustainable Agriculture. In: F.J. de Bruijn (ed). Molecular Microbial Ecology of the Rhizosphere, vol 2. Wiley Blackwell, Hoboken, New Jersey, USA, pp: 615-628.

Calvo-Polanco, M., Sánchez-Romera, B., Aroca, R. 2013. Arbuscular mycorrhizal fungi and the tolerance of plants to drought and salinity. In: R. Aroca R. (ed). Symbiotic Endophytes. SpringerVerlag, Berlin, Heidelberg, pp: 271-288.
Carvalhais, L.C., Dennis, P.G., Tyson, G.W., Schenk, P.M. 2013. Rhizosphere metatranscriptomics: challenges and opportunities. In: F.J. de Bruijn (ed). Molecular Microbial Ecology of the Rhizosphere, vol 2. Wiley Blackwell, Hoboken, New Jersey, USA, pp: 1137-1144.

Chauhan, P.S., Chaudhry, V., Mishra, S., Mishra, A., Nautiyal, C.S. 2013. Unraveling the shed of unexplored rhizosphere microbial diversity. In: F.J. de Bruijn (ed). Molecular Microbial Ecology of the Rhizosphere, vol 1. Wiley Blackwell, Hoboken, New Jersey, USA., pp: 105-114.

Chaumont, F., Tyerman, S.D. 2014. Aquaporins: highly regulated channels controlling plant water relations. Plant Physiol. 164, 1600-1618.

Cornejo, P., Meier, S., Borie, G., Rillig, M., Borie, F. 2008. Glomalin-related soil protein in a Mediterranean ecosystem affected by a copper smelter and its contribution to $\mathrm{Cu}$ and $\mathrm{Zn}$ sequestration. Sci. Total Environ. 406, 154-160.

de Bruijn, F.J. 2015. Biological nitrogen fixation. In: B. Lugtenberg (ed). Principles of Plant-Microbe Interactions. Springer International Publishing Switzerland, Heidelberg, pp: 215-224.

Dimkpa, C., Weinand, T., Asch, F. 2009. Plantrhizobacteria interactions alleviate abiotic stress conditions. Plant Cell Environ. 32, 1682-1694.

Dodd, I.C., Perez-Alfocea, F. 2012. Microbial amelioration of crop salinity stress. J. Exp. Bot. 63, 3415-3428.

Drogue, B., Combes-Meynet, E., Moënne-Loccoz, Y., Wisniewski-Dyé, F., Prigent-Combaret, C. 2013. Control of the cooperation between plant growth-promoting rhizobacteria and crops by rhizosphere signals. In: F.J. de Bruijn (ed). Molecular Microbial Ecology of the Rhizosphere, vol 1. Wiley Blackwell, Hoboken, New Jersey, USA, pp: 281-293. 
Duarte, C.M., Alonso, S., Benito, G., Dachs, J., Fernández Ríos, A.d.1.A., Montes, C., Pardo, M., Simó, R., Valladares, F., 2006. Cambio global: Impacto de la actividad humana sobre el sistema Tierra. Consejo Superior de Investigaciones Científicas (CSIC),

Ferrol, N., González-Guerrero, M., Valderas, A., Benabdellah, K., Azcón-Aguilar, C. 2009. Survival strategies of arbuscular mycorrhizal fungi in $\mathrm{Cu}$-polluted environments. Phytochem. Rev. 8, 551-559.

Franken, P., Waschke, A., Requena, N. 2012. Molecular approaches to arbuscular mycorrhiza functioning. In: B. Hock (ed). The Mycota, a comprehensive treatise on fungi as experimental systems for basic and applied research. Springer, Berlin, Heidelberg, Berlin, Heidelberg, pp: 22-37.

Germaine, K.J., McGuinness, M., Dowling, D.N. 2013. Improving phytoremediation through plant-associated bacteria. In: F.J. de Bruijn (ed). Molecular Microbial Ecology of the Rhizosphere, vol 2. Wiley Blackwell, Hoboken, New Jersey, USA, pp: 963-973.

Gianinazzi-Pearson, V., van Tuinen, D., Wipf, D.,Dumas-Gaudot, E., Recorbet, G., Lyu, Y. Doidy, J., Redecker, D., Ferrol, N. 2012. Exploring the genome of Glomeromycotan fungi. In: B. Hock (ed). The Mycota, a comprehensive treatise on fungi as experimental systems for basic and applied research. Springer, Berlin, Heidelberg, Berlin, Heidelberg, pp: 1-21.

González-Guerrero, M., Benabdellah, K., Ferrol, N., Azcón-Aguilar, C. 2009. Mechanisms underlying heavy metal tolerance in arbuscular mycorrhizas. In: C. Azcón-Aguilar, J.M. Barea, S.Gianinazzi, V. Gianinazzi-Pearson (eds). Mycorrhizas Functional Processes and Ecological Impact. Springer-Verlag, Berlin, Heidelberg, pp: 107-122.
González-Guerrero, M., Benabdellah, K., Valderas, A., Azcón-Aguilar, C., Ferrol, N. 2010. GintABC1 encodes a putative ABC transporter of the MRP subfamily induced by $\mathrm{Cu}, \mathrm{Cd}$, and oxidative stress in Glomus intraradices. Mycorrhiza 20, 137-146.

Groppa, M.D., Benavides, M.P., Zawoznik, M.S. 2012. Root hydraulic conductance, aquaporins and plant growth promoting microorganisms: A revision. Appl. Soil Ecol. 61, 247-254.

Hardoim, P. R. van Elsas, J. D. 2013. Properties of bacterial endophytes leading to maximized host fitness. In: F.J. de Bruijn (ed). Molecular Microbial Ecology of the Rhizosphere, vol 1. Wiley Blackwell, Hoboken, New Jersey, USA, pp: 405-411.

Hirsch, P.R., Mauchline, T.H., Clark, I.M. 2013a. Culture-independent molecular approaches to microbial ecology in soil and the rhizosphere. In: F.J. de Bruijn (ed). Molecular Microbial Ecology of the Rhizosphere, vol 1. Wiley Blackwell, Hoboken, New Jersey, USA, pp: 45-55.

Hirsch, P.R., Miller, A.J., Dennis, P.G. 2013b. Do root exudates exert more influence on rhizosphere bacterial community structure than other rhizodeposits? In: F.J. de Bruijn (ed). Molecular Microbial Ecology of the Rhizosphere, vol 1. Wiley Blackwell, Hoboken, New Jersey, USA, pp: 229-242.

Jayaraman, D., Forshey, K.L., Grimsrud, P.A., Ane, J.-M. 2012. Leveraging proteomics to understand plant-microbe interactions. Front. Plant Sci. 3, 44.

Jeffries, P., Barea, J.M. 2012. Arbuscular Mycorrhiza - a key component of sustainable plant-soil ecosystems. In: B. Hock (ed). The Mycota, a comprehensive treatise on fungi as experimental systems for basic and applied research. Springer, Berlin, Heidelberg, Berlin, Heidelberg, pp: 51-75. 
Jeffries, P., Gianinazzi, S., Perotto, S., Turnau, K., Barea, J.M. 2003. The contribution of arbuscular mycorrhizal fungi in sustainable maintenance of plant health and soil fertility. Biol. Fert. Soils 37, 1-16.

Jung, S., Martínez-Medina, A., López-Ráez, J.A., Pozo, M.J. 2012. Mycorrhiza-induced resistance and priming of plant defences. J. Chem. Ecol. 38, 651-664.

Kamilova, F., Okon, Y., de Weert, S., Hora, K. 2015. Commercialization of microbes: manufacturing, inoculation, best practice for objective field testing, and registration. In: B. Lugtenberg (ed). Principles of Plant-Microbe Interactions. Springer International Publishing Switzerland, Heidelberg, pp: 319-327.

Li, G., Santoni, V., Maurel, C. 2014. Plant aquaporins: Roles in plant physiology. Bioch. Et Biophys. Acta-Gen. Subj. 1840, 1574-1582.

López-Ráez, J.A., Bouwmeester, H., Pozo, M.J. 2012. Communication in the rhizosphere, a target for pest management In: E. Lichtfouse (ed). Sustainable Agriculture Reviews vol 8 . Agroecology and Strategies for Climate Change, vol 8. Sustainable Agriculture Reviews. Springer Netherlands, pp: 109-133.

Lugtenberg, B. 2015. Life of microbes in the rhizosphere. In: B. Lugtenberg (ed). Principles of Plant-Microbe Interactions. Springer International Publishing Switzerland, Heidelberg, pp: 7-15.

Malfanova, N., Lugtenberg, B.J.J., Berg, G. 2013. Bacterial endophytes: who and where, and what are they doing there? In: F.J. de Bruijn (ed). Molecular Microbial Ecology of the Rhizosphere, vol 1. Wiley Blackwell, Hoboken, New Jersey, USA, pp: 393-403.
Martinez-Viveros, O., Jorquera, M.A., Crowley, D.E., Gajardo, G., Mora, M.L. 2010. Mechanisms and practical considerations involved in plant growth promotion by rhizobacteria. J. Soil Sci. Plant Nutr. 10, 293-319.

Medina, A., Azcón, R. 2010. Effectiveness of the application of arbuscular mycorrhiza fungi and organic amendments to improve soil quality and plant performance under stress conditions. J. Soil Sci. Plant Nutr. 10, 354-372.

Mercado-Blanco, J. 2015. Life of microbes inside the plant. In: B. Lugtenberg (ed). Principles of PlantMicrobe Interactions. Springer International Publishing Switzerland, Heidelberg, pp: 25-32.

Normand, P., Queiroux, C., Tisa, L.S., Benson, D.R., Rouy, Z., Cruveiller, S., Medigue, C. 2007. Exploring the genomes of Frankia. Physiol. Plant. 130, 331-343.

Oldroyd, G.E.D., Dixon, R. 2014. Biotechnological solutions to the nitrogen problem. Curr. Opin. Biotechnol. 26, 19-24.

Olivares, J., Bedmar, E.J., Sanjuan, J. 2013. Biological nitrogen fixation in the context of global change. Mol. Plant-Microbe Interact. 26, 486-494.

Ortiz-Castro, R.,López-Bucio, J.2013. Smallmolecules involved in transkingdom communication between plants and rhizobacteria. In: F.J. de Bruijn (ed). Molecular Microbial Ecology of the Rhizosphere, vol 1. Wiley Blackwell, Hoboken, New Jersey, USA, pp: 295-307.

Pangesti, N., Pineda, A., Pieterse, C.M.J., Dicke, M., van Loon, J.J.A. 2013. Two-way plant-mediated interactions between root-associated microbes and insects: from ecology to mechanisms. Front. Plant Sci. 4. 
Pongrac, P., Vogel-Mikus, K., Poschenrieder, C., Barceló, J., Tolrà, R., Regvar, M. 2013a. Arbuscular mycorrhiza in glucosinolate-containing plants: the story of the metal hyperaccumulator Noccaea (Thlaspi) praecox (Brassicaceae). In: F.J. de Bruijn (ed). Molecular Microbial Ecology of the Rhizosphere, vol 2. Wiley Blackwell, Hoboken, New Jersey, USA, pp: 1023-1032.

Porcel, R., Aroca, R., Ruiz-Lozano, J. M.2012. Salinity stress alleviation using arbuscular mycorrhizal fungi. A review. Agron. Sustain. Dev. 32, 181-200.

Porras-Alfaro, A., Bayman, P. 2011. Hidden fungi, emergent properties: Endophytes and microbiomes. Ann. Rev. Phytopathol. 49, 291315.

Pozo, M., López-Ráez, J., Azcón-Aguilar, C., GarcíaGarrido, J. 2015. Phytohormones as integrators of environmental signals in the regulation of mycorrhizal symbioses. New Phytol. 205,14311436.

Pozo, M.J., Azcón-Aguilar, C. 2007. Unraveling mycorrhiza-induced resistance. Curr. Opin. Plant Biol. 10, 393-398.

Pozo, M.J., Jung, S.C., Martínez-Medina, A., LópezRáez, J.A., Azcón-Aguilar, C., Barea, J.M. 2013. Root allies: Arbuscular mycorrhizal fungi help plants to cope with biotic stresses. In: R. Aroca (ed). Symbiotic Endophytes. Springer-Verlag, Berlin Heidelberg, pp: 289-307.

Raaijmakers, J.M. 2015. The minimal rhizosphere microbiome. In: B. Lugtenberg (ed). Principles of Plant-Microbe Interactions. Springer International Publishing Switzerland, Heidelberg, pp: 411-417.

Raaijmakers, J.M., Lugtenberg, B.J.J. 2013. Perspectives for rhizosphere research. In: F.J. de Bruijn (ed). Molecular Microbial Ecology of the Rhizosphere, vol 2. Wiley Blackwell, Hoboken, New Jersey, USA, pp: 1227-1232.
Ravensberg, W.J. 2015. Commercialisation of microbes: Present situation and future prospects. In: B. Lugtenberg (ed). Principles of Plant-Microbe Interactions. Microbes for Sustainable Agriculture. Springer International Publishing Switzerland, Heidelberg, pp: 309-317.

Richardson, A.E., Barea, J.M., McNeill, A.M., PrigentCombaret, C. 2009. Acquisition of phosphorus and nitrogen in the rhizosphere and plant growth promotion by microorganisms. Plant Soil 321, 305339.

Rogers, C., Oldroyd, G.E.D. 2014. Synthetic biology approaches to engineering the nitrogen symbiosis in cereals. J. Exp. Bot. 65, 1939-1946.

Ruíz-Lozano, J.M., Porcel, R., Azcón, C., Aroca, R. 2012a. Regulation by arbuscular mycorrhizae of the integrated physiological response to salinity in plants: new challenges in physiological and molecular studies. J. Exp. Bot. 63, 4033-4044.

Ruíz-Lozano, J.M., Porcel, R., Barzana, G., Azcón, R., Aroca, R. 2012b. Contribution of arbuscular mycorrhizal symbiosis to plant drought tolerance: state of the art. In: R. Aroca (ed). Plant Responses to Drought Stress. From Morphological to Molecular Features. Springer, Heidelberg, Germany, pp: 335-362.

Savka, M.A., Dessaux, Y., McSpadden Gardener, B.B., Mondy, S., Kohler, P.R.A., de Bruijn, F.J., Rossbach, S. 2013. The "biased rhizosphere" concept and advances in the omics era to study bacterial competitiveness and persistence in the phytosphere. In: F.J. de Bruijn (ed). Molecular Microbial Ecology of the Rhizosphere, vol 2. Wiley Blackwell, Hoboken, New Jersey, USA, pp: 1147-1161.

Schreiter, S., Eltlbany, N., Smalla, K. 2015. Microbial communities in the rhizosphere analyzed by cultivation-independent DNA-based methods. In: B. Lugtenberg (ed). Principles of Plant-Microbe Interactions. Springer International Publishing Switzerland, Heidelberg, pp: 289-298. 
Schüßler, A., Schwarzott, D., Walker, C. 2001. A new fungal phylum, the Glomeromycota, phylogeny and evolution. Mycol. Res. 105, 1413-1421.

Selosse, M., Bessis, A., Pozo, M. 2014. Microbial priming of plant and animal immunity: symbionts as developmental signals. Trends Microbiol. 22, 607-613.

Singh, S., Srivastava, K. Sharma, S., Sharma. A.K. 2014. Mycorrhizal inoculum production. In: Z. M.Solaiman, L. K.Abbott, A. Varma. Mycorrhizal fungi: use in sustainable agriculture and land restoration. Soil Biology vol. 41. Springer-Verlag, Berlin, Heidelberg, pp: 67-80.

Smith, S.E., Read, D.J., 2008. Mycorrhizal Symbiosis. 3rd edn. Elsevier, Academic Press, New York.

Spence, C., Bais, H. 2013. Probiotics for plants: rhizospheric microbiome and plant fitness. In: F.J. de Bruijn (ed). Molecular Microbial Ecology of the Rhizosphere, vol 2. Wiley Blackwell, Hoboken, New Jersey, USA, pp: 713-721.

Tamayo, E., Gómez-Gallego, T., Azcón-Aguilar, C., Ferrol, N. 2014. Genome-wide analysis of copper, iron and zinc transporters in the arbuscular mycorrhizal fungus Rhizophagus irregularis. Front. Plant Sci. 5, 547.

van der Heijden, M. G. A., Martin, F. M., Selosse, M. A., Sanders, I. R. 2015. Mycorrhizal ecology and evolution: the past, the present, and the future. New Phytol. 205, 1406-1423.
Venkateshwaran, M. 2015. Exploring the feasibility of transferring nitrogen fixation to cereal crops. In: B. Lugtenberg (ed). Principles of Plant-Microbe Interactions. Springer International Publishing Switzerland, Heidelberg, pp: 403-410.

Vitousek, P.M., Mooney, H.A., Lubchenco, J., Melillo, J.M. 1997. Human domination of Earth's ecosystems. Science 277, 494-499.

Zancarini, A., Lépinay, C., Burstin, J., Duc, G., Lemanceau, P., Moreau, D., Munier-Jolain, N., Pivato, B., Rigaud, T., Salon, C., Mougel, C. 2013. Combining molecular microbial ecology with ecophysiology and plant genetics for a better understanding of plant-microbial communities' interactions in the rhizosphere. In: F.J. de Bruijn (ed). Molecular Microbial Ecology of the Rhizosphere, vol 1. Wiley Blackwell, Hoboken, New Jersey, USA, pp: 69-86.

Zolla, G., Bakker, M.G., Badri, D.V., Chaparro, J.M., Sheflin, A.M., Manter, D.K., Vivanco, J. 2013. Understanding root-microbiome interactions. In: F.J. de Bruijn (ed). Molecular Microbial Ecology of the Rhizosphere, vol 2. Wiley Blackwell, pp: 745-754. 